\title{
Comparative Study of the Moss Flora of Ismailia Governorate with Other Territories of Egypt
}

\author{
Yasmin M. Hassan ${ }^{1 *}$, Usama Y. Abou-Salama ${ }^{2}$, Wafaa M. Kamel ${ }^{1}$, Elsayeda M. Gamal Eldin ${ }^{1}$ \\ ${ }^{1 .}$ Botany Department, Faculty of Science, Suez Canal University, Ismailia, Egypt \\ ${ }^{2}$ Botany Department, Faculty of Science, Ain Shams University, Cairo, Egypt
}

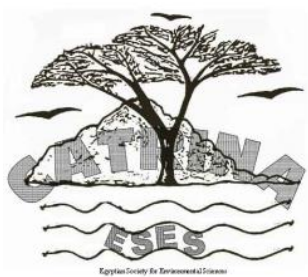

\section{ABSTRACT}

This study was conducted to held comparisons between moss flora of Ismailia governorate and the different territories in Egypt. The last study done on this governorate was carried out three decades before. Fifteen excursions were set out for this purpose. Three hundred and ten moss samples were collected from Ismailia governorate. The study revealed that floral similarity was largest between Ismailia governorate and the Southern Sinai massive (S). On the other hand, both Nile Nubia (Nn) and Gebel Elba (GE) showed the lowest species similarity in comparison with the study area Keywords: Moss, Flora, Ismailia, Territories, Egypt.

\section{INTRODUCTION}

Bryological work in Egypt has been concerned mainly with the enumeration and description of moss species encountered during excursions of researchers in different regions of the country. El-Saadawi and colleagues published a large number of papers including six important checklists: El-Saadawi and Badawi (1977), El-Saadawi et al. (1986), El-Saadawi et al. (1999), El-Saadawi et al. (2003), El-Saadawi et al. (2013) and El-Saadawi et al. (2015). All the previous studies until 2015 showed that the total number of moss taxa reported from Egypt is 181 and is classified into 56 genera, 17 families and 10 orders.

The Ismailia governorate is located in the eastern part of Egypt at the middle part of Suez Canal. It is a part of the East Nile Delta region (Nd), Galala Desert (Dg) and Isthmic Desert (Di). It is bounded at the east by North Sinai and Suez Canal (that penetrates Temsah Lake and Bitter Lake), Sharkia governorate at the west, PortSaid governorate at the north and Suez governorate at the south. The Ismailia governorate is located between $31^{\circ}$ $40^{\prime}$ to $32^{\circ} 40^{\prime}$ E Longitude, and $30^{\circ} 15^{\prime}$ to $30^{\circ} 57^{\prime} \mathrm{N}$ Latitude (Map 1). The total area of Ismailia governorate is about $5,067 \mathrm{~km}^{2}$ and consists of seven regions (marākiz). These are Ismailia, Fayed, El Tal El Kabier, Abou Sweir and El Kassaseen, East Kantara and West Kantara.

According to the Köppen-Geiger classification (BWh) and the Holdridge life zones system of bioclimatic classification, Ismailia governorate has a subtropical desert / low-latitude arid hot climate. Also it is situated in or near the subtropical desert biome (http://www.ismailia.climatemps.com).

Ismailia governorate is located mainly in Galala desert. Since 1972, only three studies of mosses had been done from Galala desert. These are Imam and Ghabbour (1972), Lashin (1990) and Shabbara (2006). They recognized 30 species belonging to 14 genera (including genus Leptobarbula Schimp. which was recorded only in this territory), 5 families and 4 orders. Lashin (1990) in his study on the bryoflora of Suez Canal Region reported 29 moss taxa, out of these 26 taxa identified to the specific level and 3 entities only to generic level (Barbula sp , Semibarbula sp and Funaria sp).

The present study aims to held comparisons between moss flora of Ismailia governorate and the different territories in Egypt. This will help to draw conclusions about various factors that affect the distribution of moss species across Egypt.

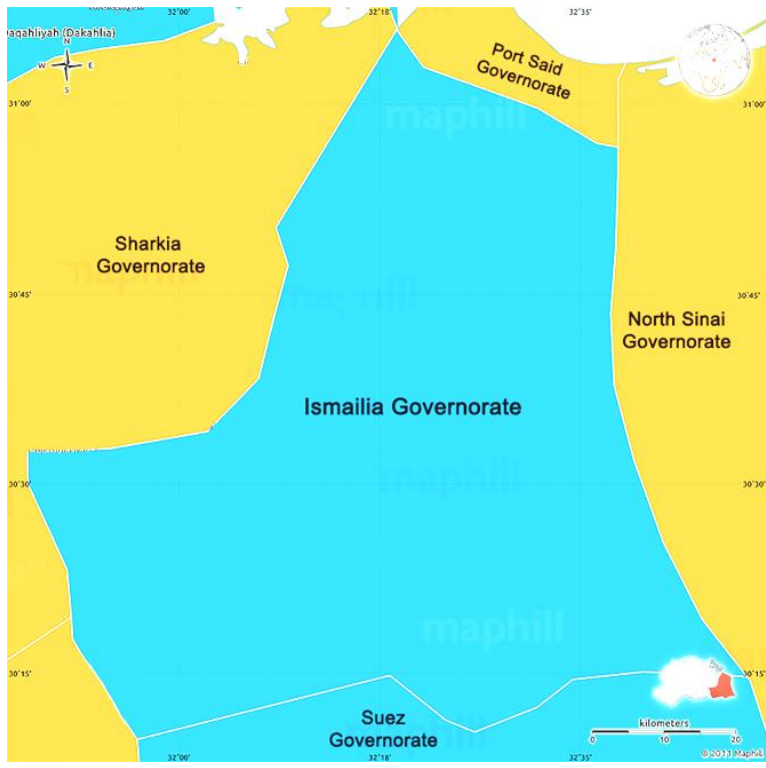

Map (1): The location of Ismailia governorate.

\section{MATERIALS AND METHODS}

To achieve the aim of the present study, three hundred and ten samples were collected during 15 excursions covering the period from November 2014 to August 2016 from cities, marākiz, villages, sub-villages and ezbets in Ismailia governorate. Mosses were mostly collected from shaded and sunny places borders of irrigation canals, water wheels, water reservoirs, water basins and walls of bridges. They occurred mainly on 


\section{Comparative Study of the Moss Flora}

red bricks, walls covered by cement and/or mud.

Specimens were examined, sectioned according to Abou Salama (1985) and Wilson (1990). The studied specimens were identified by being matched with the pre-identified Egyptian moss specimens from different phytogeographrical territories of Egypt. In addition, moss floras of different countries of the world were used. Adaptation to diverse habitats was taken into consideration in determining precise identification. Detailed information about all collected samples were available in herbarium packets kept at the Herbarium of the Suez Canal University (SCU-I) Ismailia, Faculty of Science, Botany Department (Appendix 1).

\section{RESULTS}

The updated species list of the moss flora of Ismailia governorate includes 29 species. These species belong to 13 genera, 4 families and 4 orders. The present study according to El-Saadawi et al. (2015) added Bryoerythrophyllum and Oxystegus as a new genera to the moss flora of Egypt and added 3 species as new records to Egypt namely; Bryoerythrophyllum inaeq-ualifolium, Oxystegus tenuirostris and Trichostomum planifolium, raising the number of mosses recorded in Egypt to 184 species.

It is obvious from the distribution of the studied species (Table 1 and Figure 1) that, Barbula unguiculata had the highest percentage of occurrence of all recorded species in the study area, followed by Funaria hygrometrica. Abou Sweir region is characterized by high diversity due to the presence of 18 species, followed by Ismailia region and El Tal El Kabier region (11 species each). This is due to favourable climatic conditions, good soil with low salinity and the first start of Ismailia canal as the fresh water source. But East Kantara region was poor in mosses ( 6 species) due to the high salinity and gypsum soil apparently hinder the growth of mosses.

Table (1): Distribution of the 29 studied moss species in the study area and number of their specimens.

\begin{tabular}{|c|c|c|c|}
\hline Recorded Species & Region & $\begin{array}{c}\text { No. of } \\
\text { specimens }\end{array}$ & Total \\
\hline Fissidens bryoides & Fayed & 2 & 2 \\
\hline Barbula bolleana & El Kassaseen & 7 & 7 \\
\hline B. convoluta & Abou Sweir & 3 & 3 \\
\hline \multirow{4}{*}{ B. indica } & El Kassaseen & 11 & \multirow{2}{*}{12} \\
\hline & West Kantara & 1 & \\
\hline & El Kassaseen & 43 & \multirow{6}{*}{99} \\
\hline & Abou Sweir & 23 & \\
\hline \multirow{4}{*}{ B. unguiculata } & El Tal El Kabier & 9 & \\
\hline & West Kantara & 4 & \\
\hline & Ismailia & 18 & \\
\hline & Fayed & 2 & \\
\hline \multirow[t]{2}{*}{$\begin{array}{l}\text { Bryoerythrophyllum } \\
\text { inaequalifolium }\end{array}$} & El Tal El Kabier & 3 & \multirow[t]{2}{*}{3} \\
\hline & Abou Sweir & 3 & \\
\hline \multirow[t]{4}{*}{ Didymodon fallax } & West Kantara & 2 & \multirow[t]{4}{*}{6} \\
\hline & East Kantara & 1 & \\
\hline & El Kassaseen & 5 & \\
\hline & Abou Sweir & 2 & \\
\hline \multirow[t]{3}{*}{ D. luridus } & El Tal El Kabier & 1 & \multirow[t]{3}{*}{11} \\
\hline & West Kantara & 2 & \\
\hline & Fayed & 1 & \\
\hline D. spadiceus & El Kassaseen & 4 & 4 \\
\hline D. tophaceus & El Kassaseen & 4 & 4 \\
\hline \multirow[t]{2}{*}{ D. vinealis } & Abou Sweir & 2 & \multirow{2}{*}{3} \\
\hline & East Kantara & 1 & \\
\hline \multirow[t]{2}{*}{ Gymnostomum aeruginosum } & Abou Sweir & 3 & \multirow{2}{*}{4} \\
\hline & East Kantara & 1 & \\
\hline G. calcareum & Abou Sweir & 3 & 3 \\
\hline G. viridulum & Ismailia & 1 & 1 \\
\hline \multirow[t]{2}{*}{ Splachnobryum obtusum } & El Tal El Kabier & 1 & \multirow[t]{2}{*}{1} \\
\hline & Abou Sweir & 8 & \\
\hline \multirow{5}{*}{ Oxystegus tenuirostris } & El Tal El Kabier & 2 & \multirow{5}{*}{17} \\
\hline & West Kantara & 1 & \\
\hline & Ismailia & 6 & \\
\hline & El Kassaseen & & \\
\hline & Abou Sweir & & \\
\hline \multirow[t]{3}{*}{ Trichostomum brachydontium } & El Tal El Kabier & 13 & \multirow[t]{3}{*}{20} \\
\hline & Ismailia & & \\
\hline & Fayed & & \\
\hline \multirow{2}{*}{ T. crispulum } & El Kassaseen & 2 & \multirow{2}{*}{5} \\
\hline & Fayed & 3 & \\
\hline
\end{tabular}


Hassan et al.

\begin{tabular}{|c|c|c|c|}
\hline Recorded Species & Region & $\begin{array}{c}\text { No. of } \\
\text { specimens }\end{array}$ & Total \\
\hline \multirow{6}{*}{ T. planifolium } & Abou Sweir & 7 & \multirow{6}{*}{26} \\
\hline & El Tal El Kabier & 5 & \\
\hline & West Kantara & 3 & \\
\hline & Ismailia & 5 & \\
\hline & Fayed & 5 & \\
\hline & East Kantara & 1 & \\
\hline \multirow{3}{*}{ Entosthodon fascicularis } & Abou Sweir & 7 & \multirow{2}{*}{8} \\
\hline & Ismailia & 1 & \\
\hline & Abou Sweir & 34 & \multirow{6}{*}{45} \\
\hline \multirow{5}{*}{ Funaria hygrometrica } & El Tal El Kabier & 4 & \\
\hline & West Kantara & 2 & \\
\hline & Ismailia & 3 & \\
\hline & Fayed & 1 & \\
\hline & East Kantara & 1 & \\
\hline \multirow{2}{*}{ Bryum argenteum } & Abou Sweir & 1 & \multirow{2}{*}{5} \\
\hline & Ismailia & 4 & \\
\hline B. funkii & Abou Sweir & 3 & 3 \\
\hline \multirow[t]{3}{*}{ B. gemmiparum } & Abou Sweir & 18 & \multirow[t]{3}{*}{18} \\
\hline & El Kassaseen & 3 & \\
\hline & Abou Sweir & 18 & \\
\hline \multirow[t]{4}{*}{ B. radiculosum } & El Tal El Kabier & 7 & \multirow[t]{4}{*}{40} \\
\hline & West Kantara & 4 & \\
\hline & Ismailia & 8 & \\
\hline & El Tal El Kabier & 7 & \\
\hline \multirow[t]{4}{*}{ B. subapiculatum } & West Kantara & 2 & \multirow[t]{4}{*}{10} \\
\hline & Fayed & 1 & \\
\hline & El Kassaseen & 4 & \\
\hline & Abou Sweir & 2 & \\
\hline \multirow{4}{*}{ Imbribryum alpinum } & El Tal El Kabier & 14 & \multirow{4}{*}{28} \\
\hline & West Kantara & 2 & \\
\hline & Ismailia & 5 & \\
\hline & East Kantara & 1 & \\
\hline \multirow[t]{2}{*}{ Ptychostomum imbricatulum } & Abou Sweir & 2 & \multirow[b]{2}{*}{5} \\
\hline & Ismailia & 3 & \\
\hline P. pseudotriquetrum & Fayed & 1 & 1 \\
\hline
\end{tabular}

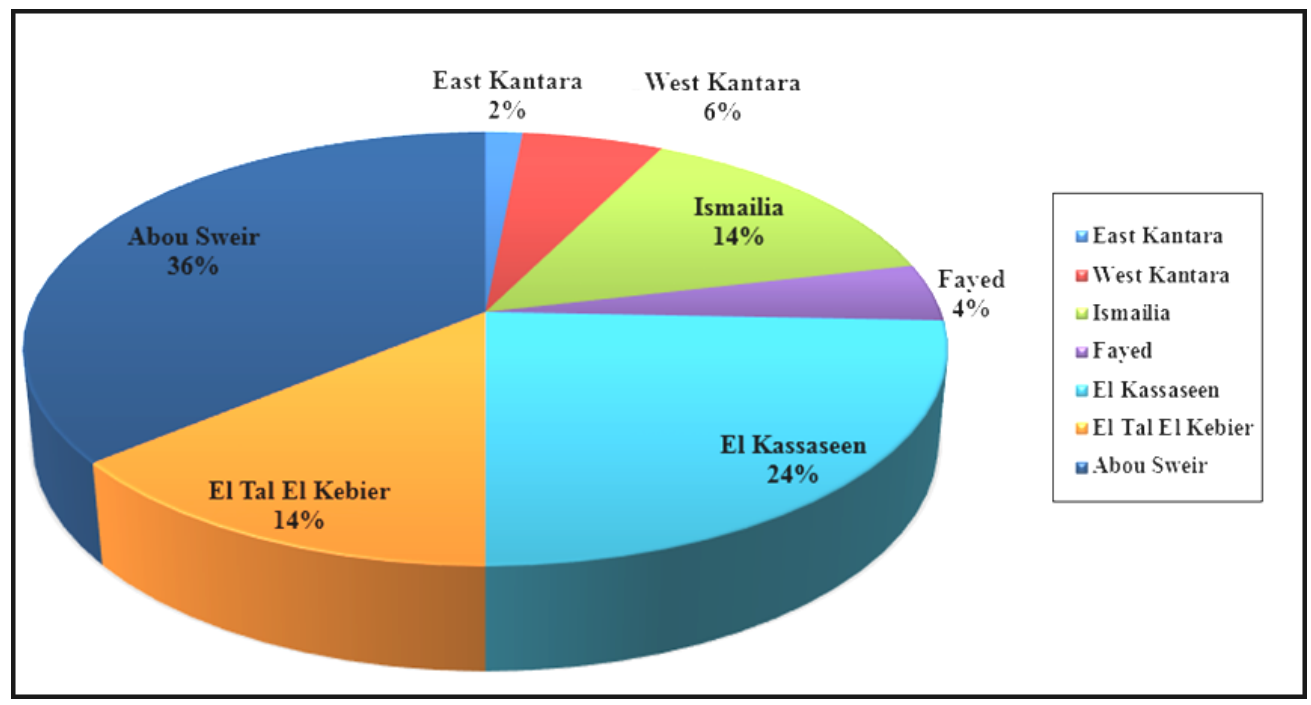

Figure (1): Percentage of occurrence of species recorded in each region.

A comparison between the species recorded in Ismailia governorate with those of other territories of Egypt (Table 2 and Map 2) show that floral similarity was largest between Ismailia governorate and the Southern Sinai massive $(\mathrm{S})$; there are 21 species common to both areas, followed by Nile Delta $(\mathrm{Nd})$ sharing 17 species. On the other hand, both Nile Nubia (Nn) and Gebel Elba (GE) showed the lowest species similarity (5 and 2 species respectively) in comparison with the study area. (El-Saadawi et al. 2015). 


\section{Comparative Study of the Moss Flora}

Table (2): Occurrence of species recorded in the present study in other territories of Egypt. (For explanation of abbreviations see map 2)

\begin{tabular}{|c|c|c|c|c|c|c|c|c|c|c|c|c|}
\hline Recorded Species in Present Study & Nn & Nv & Nd & $\mathbf{N f}$ & Cai & $\mathbf{D g}$ & Di & $\mathbf{S}$ & GE & $\mathbf{O}$ & Mma & Territories \\
\hline Fissidens bryoides & & & + & & + & + & + & & & & + & 5 \\
\hline Barbula bolleana & + & + & + & + & + & & & + & & & + & 7 \\
\hline B. convoluta & + & & + & & & + & & + & & & & 4 \\
\hline B. indica & & + & + & & + & & & & & & & 3 \\
\hline B. unguiculata & & + & + & + & + & + & & & & & + & 6 \\
\hline * Bryoerythrophyllum inaequalifolium & & & & & & & & & & & & - \\
\hline Didymodon fallax & & & + & + & & & + & + & & + & + & 6 \\
\hline D. luridus & & & + & + & + & + & & + & & & + & 6 \\
\hline D. spadiceus & & & & & & + & & + & & & & 2 \\
\hline D. tophaceus & + & + & + & + & + & + & & + & + & + & + & 10 \\
\hline D. vinealis & & & + & & & & + & + & & + & + & 5 \\
\hline Gymnostomum aeruginosum & & & + & + & + & & & + & & + & & 5 \\
\hline G. calcareum & & + & + & + & & + & & + & + & & + & 7 \\
\hline G. viridulum & & + & & & + & + & + & + & & & + & 6 \\
\hline Splachnobryum obtusum & & & + & & & & & & & & & 1 \\
\hline *Oxystegus tenuirostris & & & & & & & & & & & & - \\
\hline Trichostomum brachydontium & & & + & & & & + & & & & & 2 \\
\hline T. crispulum & & & + & & & & + & + & & & + & 4 \\
\hline *T.planifolium & & & & & & & & & & & & - \\
\hline Entosthodon fascicularis & & & & & + & & + & + & & & & 3 \\
\hline Funaria hygrometrica & + & + & + & + & + & + & + & + & & + & + & 10 \\
\hline Bryum argenteum & & & & & + & + & + & + & & + & + & 6 \\
\hline B. funkii & & & & & & + & + & + & & + & & 4 \\
\hline B. gemmiparum & & & & + & + & & & + & & + & + & 5 \\
\hline B. radiculosum & & & + & & + & & + & + & & + & + & 6 \\
\hline B. subapiculatum & & + & & & + & & & + & & + & & 4 \\
\hline Imbribryum alpinum & + & & & + & + & & & + & & & & 4 \\
\hline Ptychostomum imbricatulum & & & + & & + & + & + & + & & + & + & 7 \\
\hline P. pseudotriquetrum & & & & & & & & + & & + & & 2 \\
\hline Total Moss Species & 5 & 8 & 17 & 10 & 16 & 12 & 12 & 21 & 2 & 12 & 15 & \\
\hline
\end{tabular}

$\left.{ }^{*}\right)$ New record to Egypt.

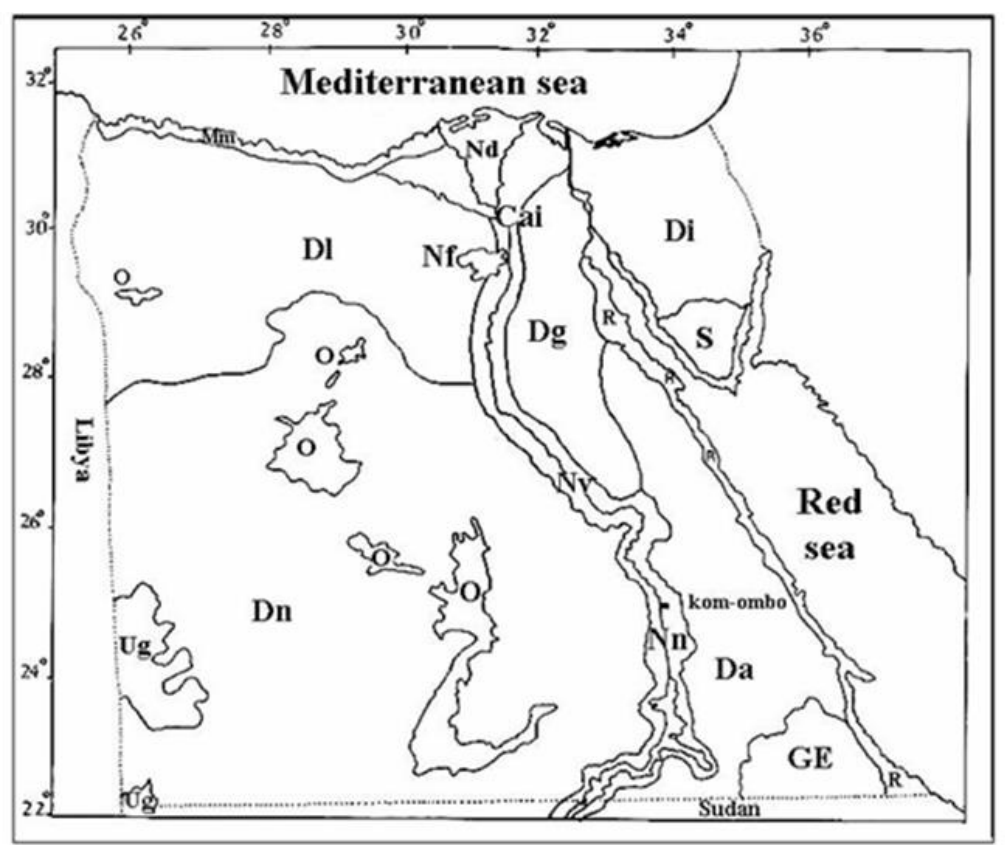

Map (2): The phytogeographical territories of Egypt (after El-Saadawi et al. 2015; with little modification). Cai: Cairo area; Da: Arabian desert; Dg: Galala Desert; Di: Isthmic Desert; Dl: Libyan Desert; Dn: Nubian Desert; GE: Gebel Elba; Mma: western Mediterranean coastal land (Mareotic sector); Nd: Nile Delta; Nf: Nile Fayoum; Nn: Nile Nubia, from Kom Ombo southwards to Egyptian boundaries with the Sudan including the areas now inundated by the waters of Lake Naser since 1965; Nv: Nile Valley, from Cairo-Giza to Kom Ombo; O: Oasis of the Nubian and Libyan Desert; R: Red Sea coastal plains; S: Southern Sinai massive (Sinai proper i.e. relatively high mountains, south of Isthmic desert); Ug: Gebel Uweinat. 


\section{DISCUSSION}

Established taxa are those widely distributed in more or less similar ecosystems in an area. Distribution of mosss pecies is mainly affected by edaphic and climatic factors especially rain and wind which carry propagules across long distances (Show and Gonffinet, 2000). A warmer and dryer climate resulting from global warming will undoubtedly cause changes in vegetation and landforms. Many bryophytes are sensitive to the increase in temperature (Dennis and Dale, 1994). The short-term effect of a simulated environmental change (increased temperature) was a decreased bryophyte growth and abundance (Jägerbrand, 2006).

Sinai and Ismailia governorate fall in the same biogeographical region (part of the Saharo-Arabian deserts) therefore affected by the same environmental factors. This explains the highest degree of floral similarity between the two regions (McGinnies et al., 1968; Danin, 1983).

Water borne propagules of mosses coming from the south and from countries of Nile basin may explain the strong relationship between moss flora of Ismailia governorate and Nile Delta (El-Saadawi et al., 2003).

On the other hand, Nile Nubia and Gebel Elba regions fall in the Sudano-Saharan biogeographical region. Thereby, those two regions showed less similarity in comparison with the study area.

\section{CONCLUSION}

Environmental factors governing the geographical distribution of moss flora in Egypt are climatic and edaphic factors.

\section{REFERENCES}

ABOU SALAMA, U.Y. 1985. Morphological Studies on some Egyptian Bryophytes in Southern Sinai. M.Sc. Thesis, Ain Shams University, Cairo, Egypt.

DANIN, A. 1983. Desert Vegetation of Israel and Sinai. Jerusalem, Israel: Cana Publishing House.
DENNIS, L., AND H. DALE. 1994. Responses of Northern Peatlands to climate change: Effects on Bryophytes. J. Hattori Bot. Lab. No; 75:119-132.

EL-SAADAWI, W., AND A. BADAWI. 1977. The moss flora of Egypt. I. Order Pottiales. Public. Cairo University Herbarium, No.7 \&8: 181-206.

EL-SAADAWI, W., W.S. ABOU EL-KHEIR, AND M.H. DARWISH. 1986. Notes on Egyptian mosses with one new record. Lindbergia 12: 129-131.

EL-SAADAWI, W.E., A. BADAWI, H.M. SHABBARA, U.Y. ABOU SALAMA, AND M. REFAI. 1999. An updated list of Egyptian mosses. Taeckholmia 19: 77-96.

EL-SAADAWI, W.E., H.M. SHABBARA, M.K. IBRAHIM, AND M.A. TAHA. 2015. An annotated checklist of Egyptian mosses. Taeckholmia 35: 1-23. http://www.ismailia.climatemps.com/(Accessed in 31/12/2016).

EL-SAADAWI, W.E., H.M. SHABBARA, S.M. REFAI, AND U.Y. ABOU SALAMA. 2003. Mosses of different phytogeographical territories of Egypt. Bocconea 16(1): 133-146.

EL-SAADAWI, W.E., U.Y. ABOU SALAMA, H.M. SHABBARA, R.M. ROS, AND V. MAZIMPAKA. 2013. Mosses of the Mediterranean, an annotated checklist. Cryptogamie Bryologie 34(2): 99-283.

IMAM, M. AND S. GHABBOUR. 1972. A contribution to the moss flora of Egypt; Botaniska Notiser, 125: 518-522.

JÄGERBRAND, A.K. 2006. Effects of Global Climate Change on Tundra Bryophytes. Botanical Society of America; 3: 235-242.

LASHIN, G.M.A.S. 1990. Studies on bryoflora Suez Canal Region. M. Sc. Thesis, Zagazig University, Zagazig, Egypt.

MCGINNIES, W.G., B.J. GOLDMAN, AND P. PAYLORE. 1968. Deserts of the World. Tucson: University of Arizona Press. pp. 788.

SHABBARA, H.M. 2006. Rare but interesting moss records from Egypt. Taeckholmia 26: 121-129.

SHOW A. J. AND GOFFINET B. 2000. Bryophyte Biology. Cambridge University. Press.

WILSON, P. 1990. How to make thin free sections. Evansia, 7 (1): 16 


\section{Comparative Study of the Moss Flora}

APPENDIX (1) :All information about samples collected from Ismailia governorate; region and site of collection, habitat, date of collection, herbarium number and GPS data, N.B. YM (Yasmin Mohamed) collector's Name, Zeer: large porcelain porous drinking pot.

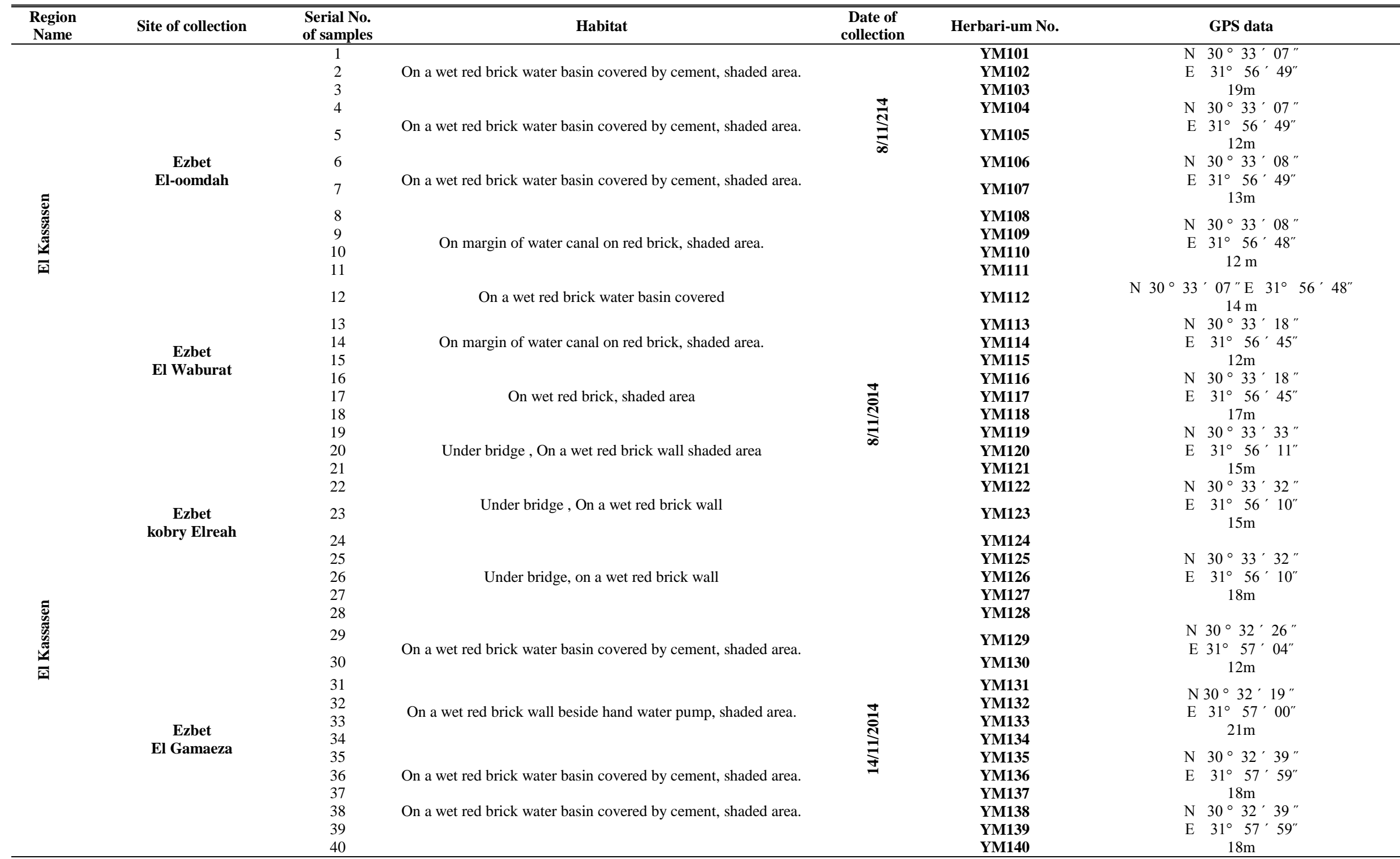


Hassan et al.

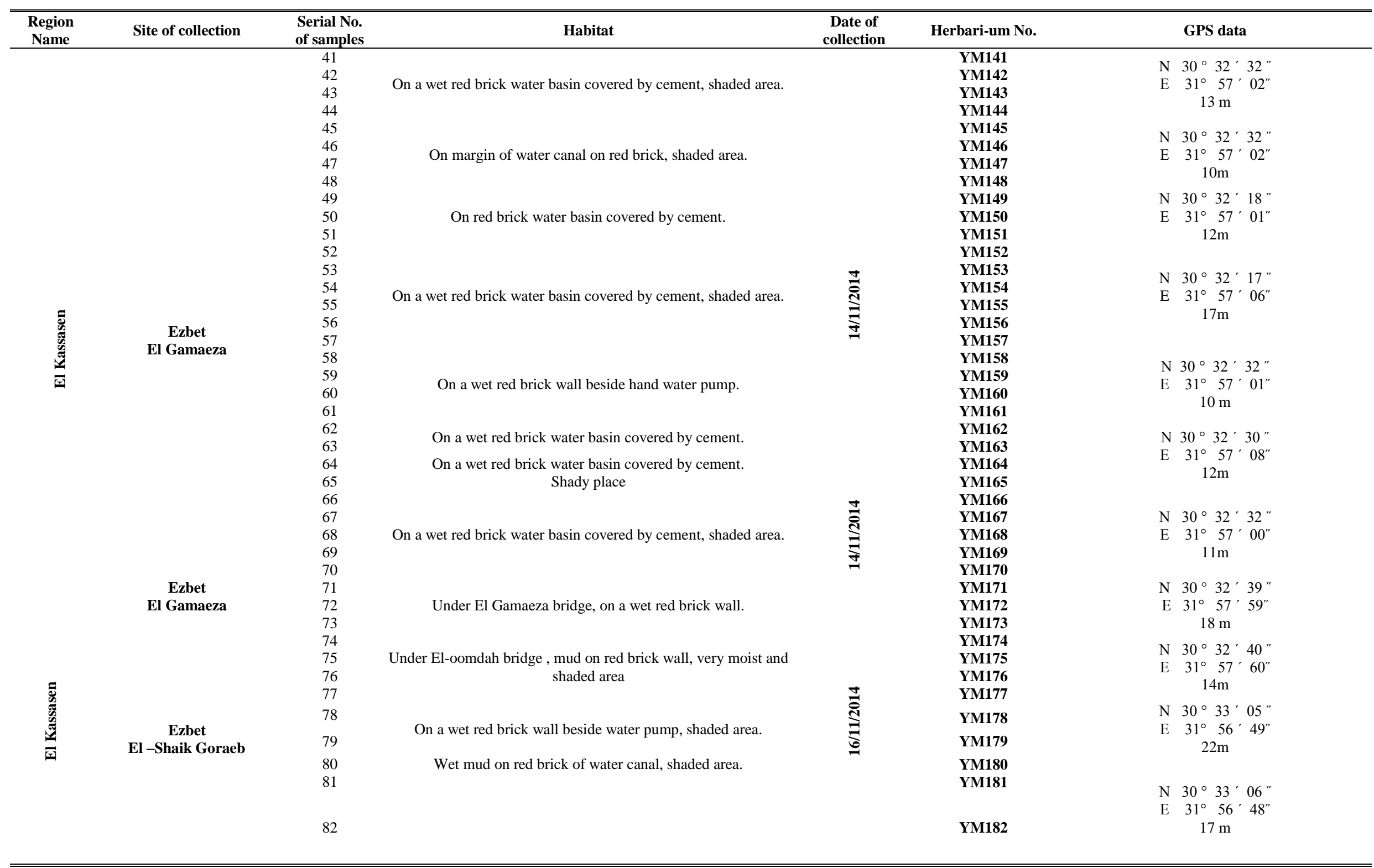




\section{Comparative Study of the Moss Flora}

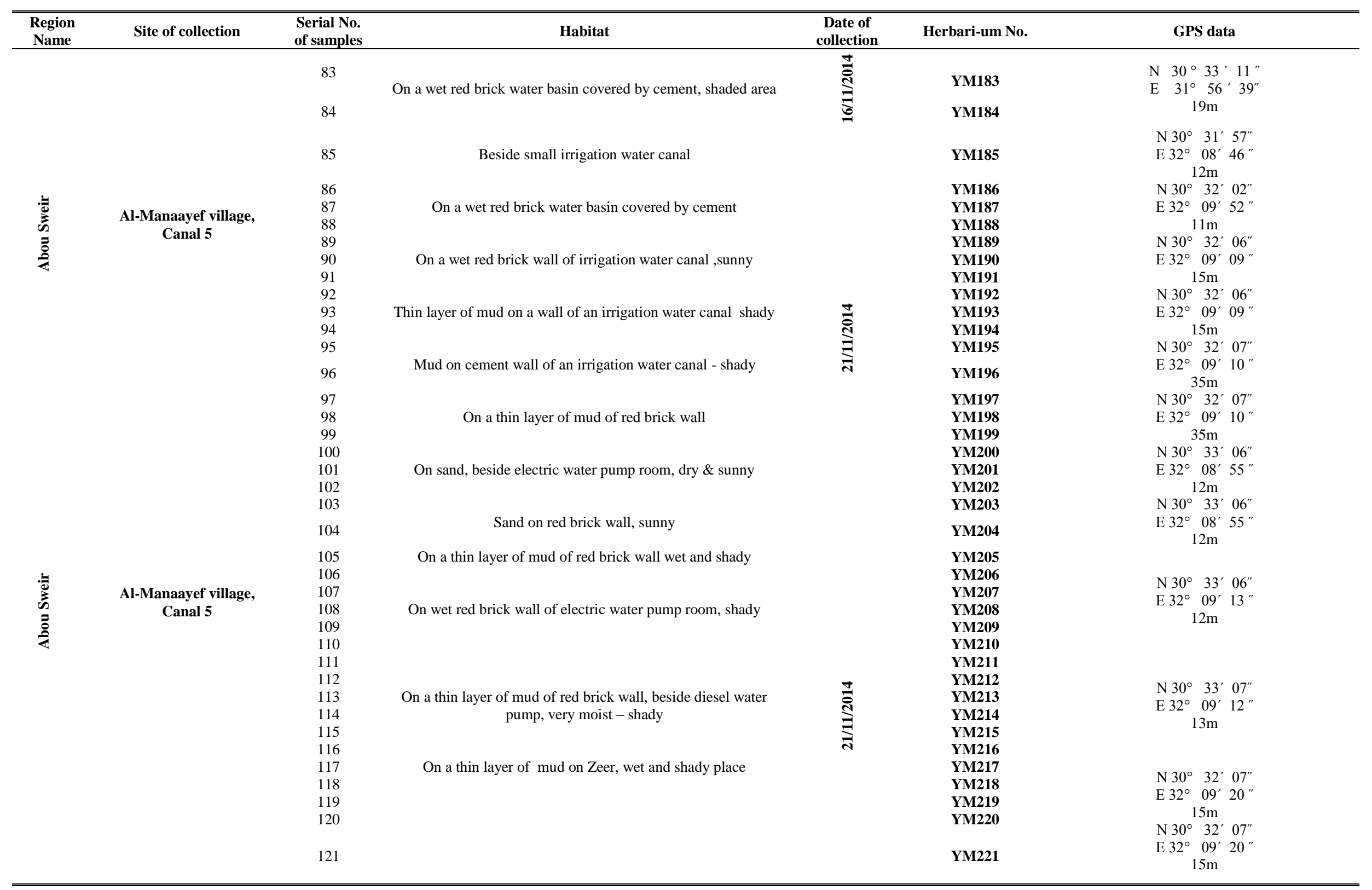




\section{Hassan et al.}

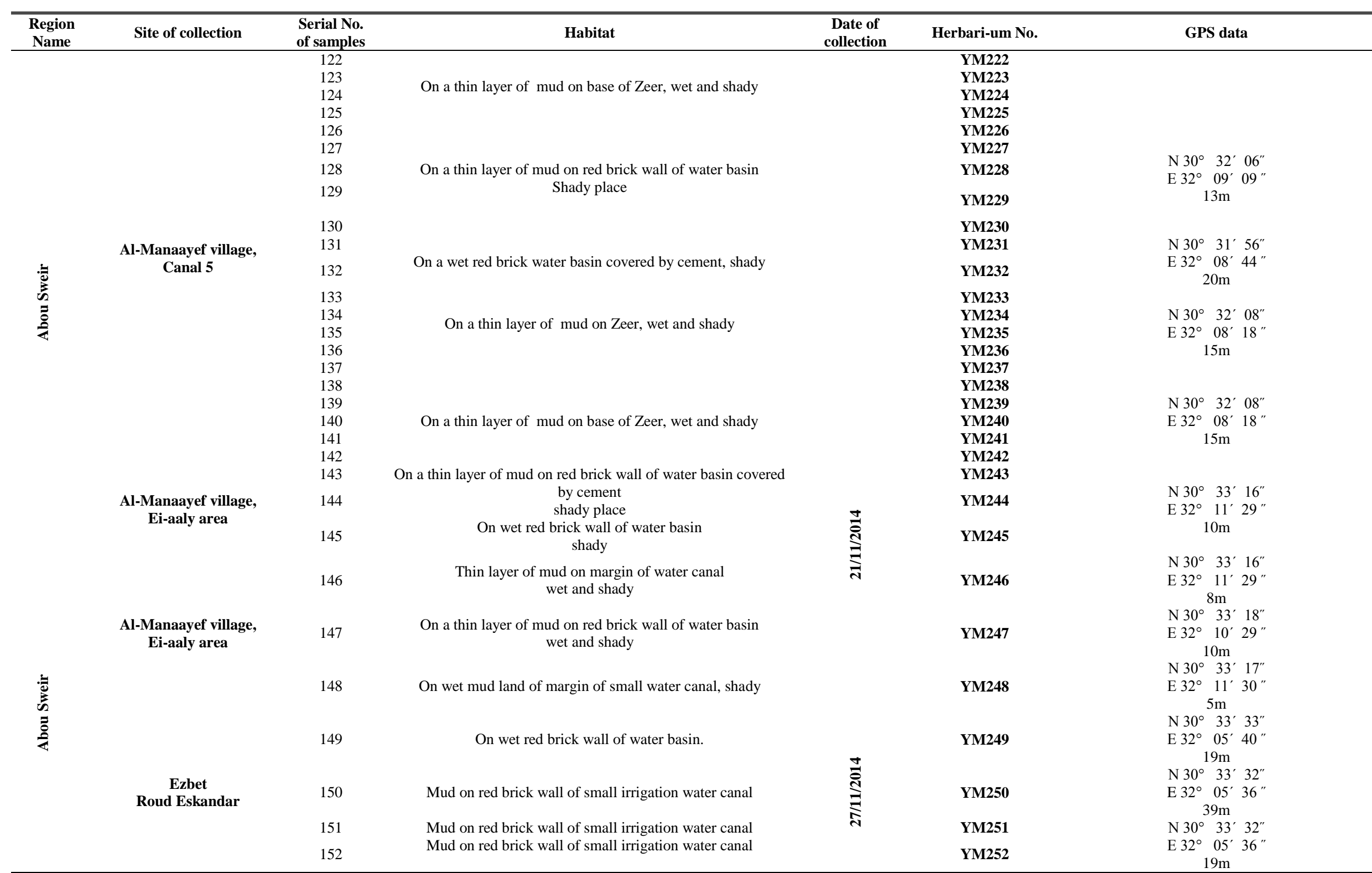




\section{Comparative Study of the Moss Flora}

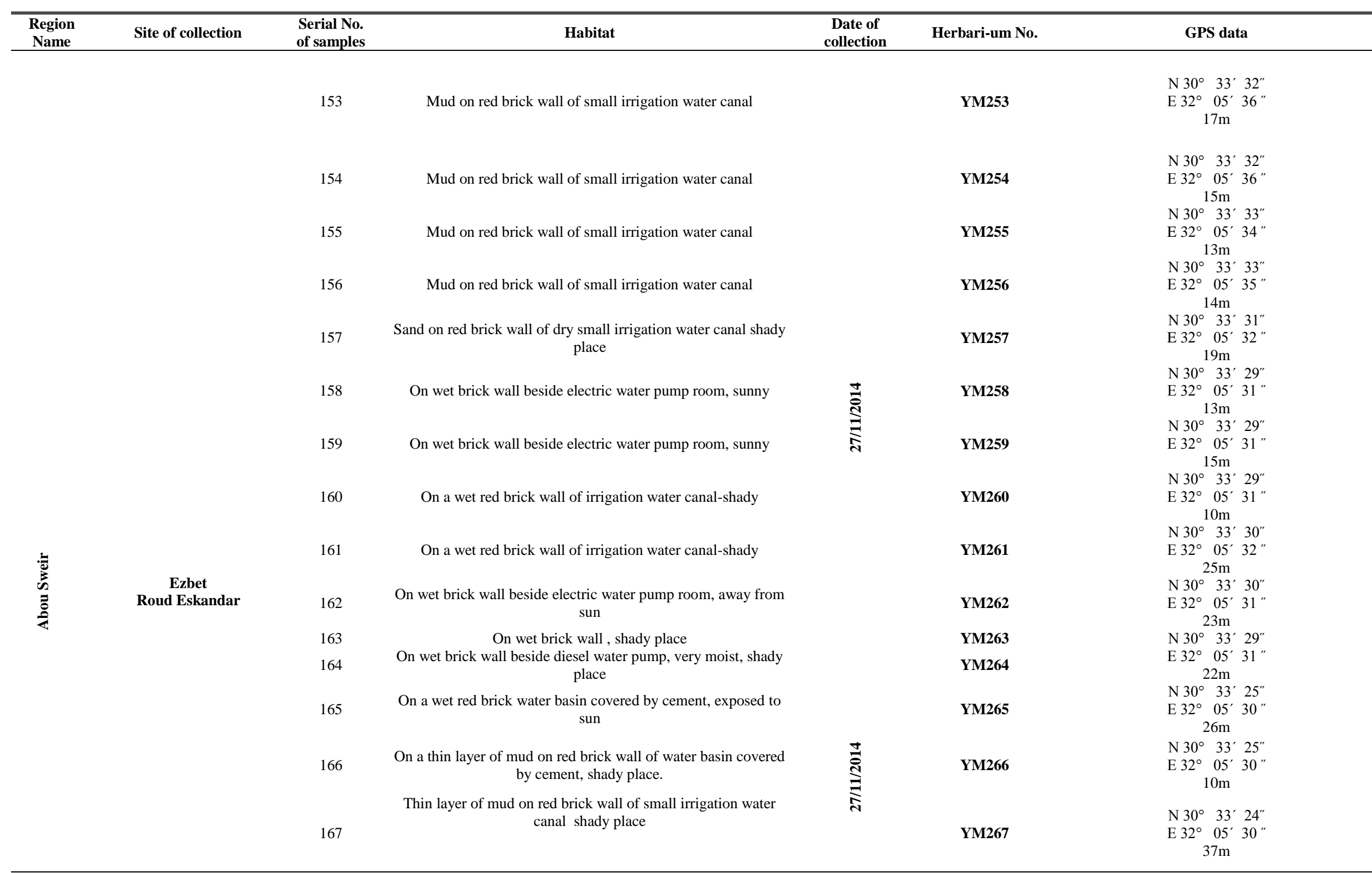




\section{Hassan et al.}

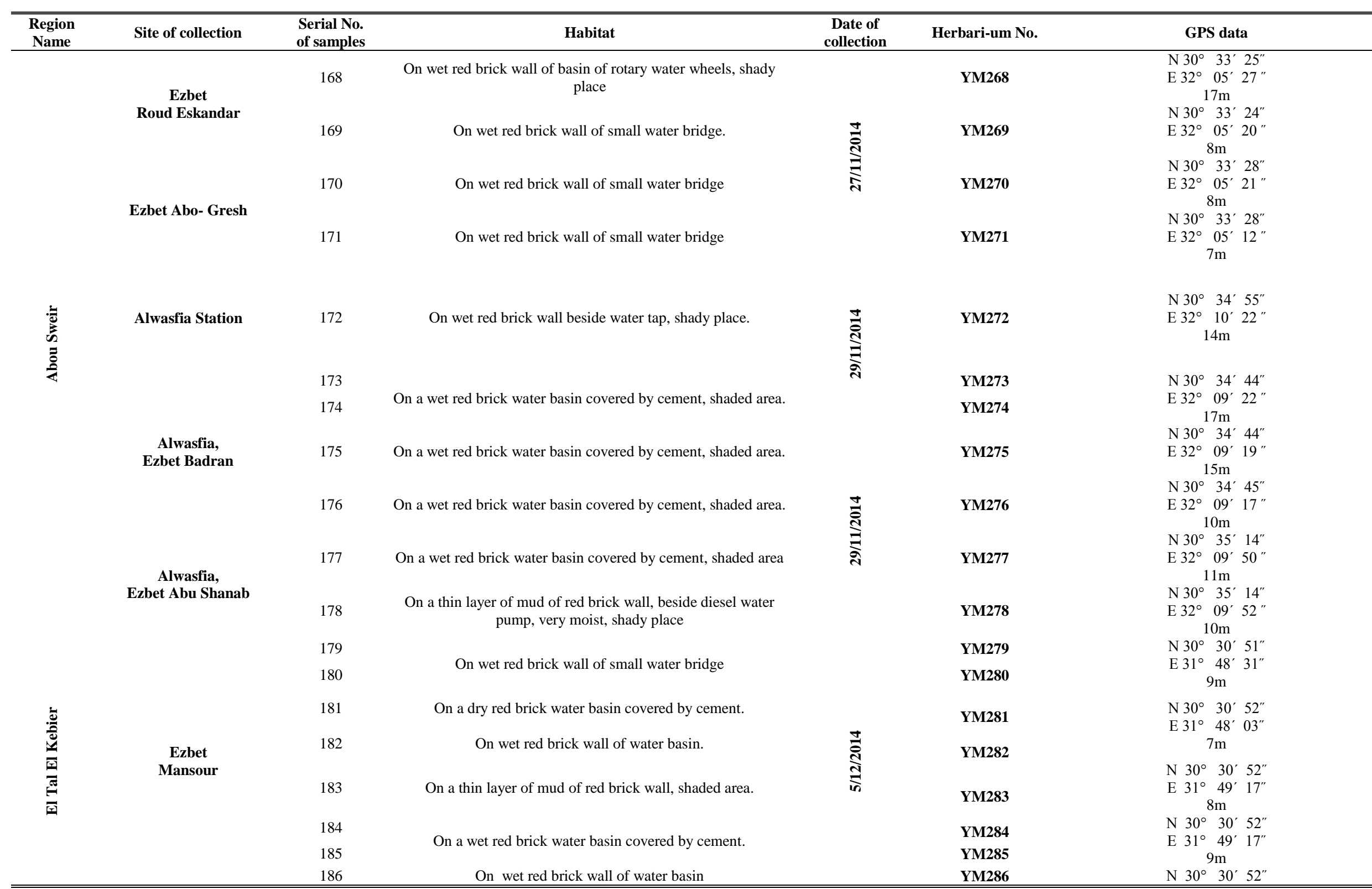




\section{Comparative Study of the Moss Flora}

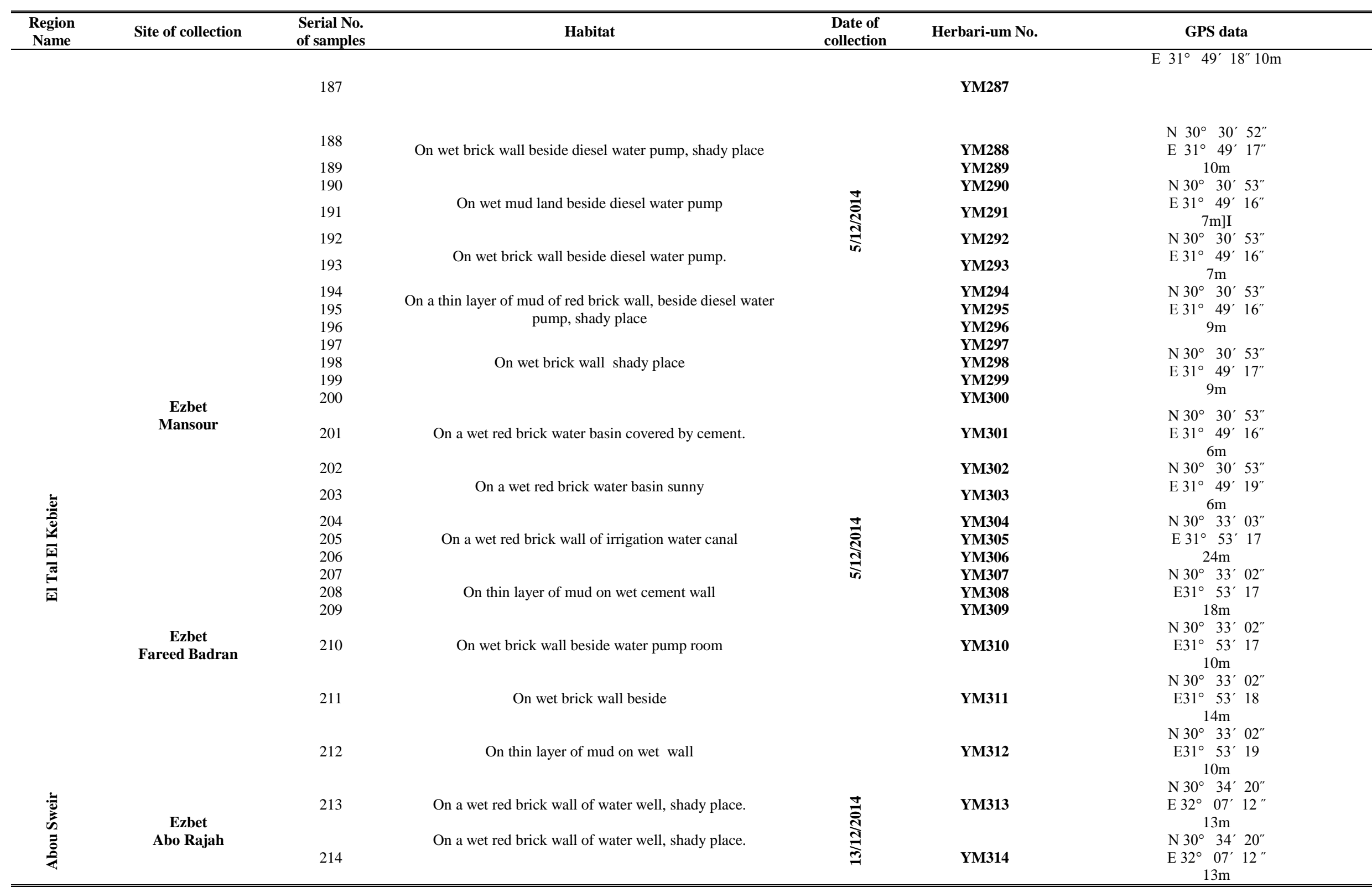




\section{Hassan et al.}

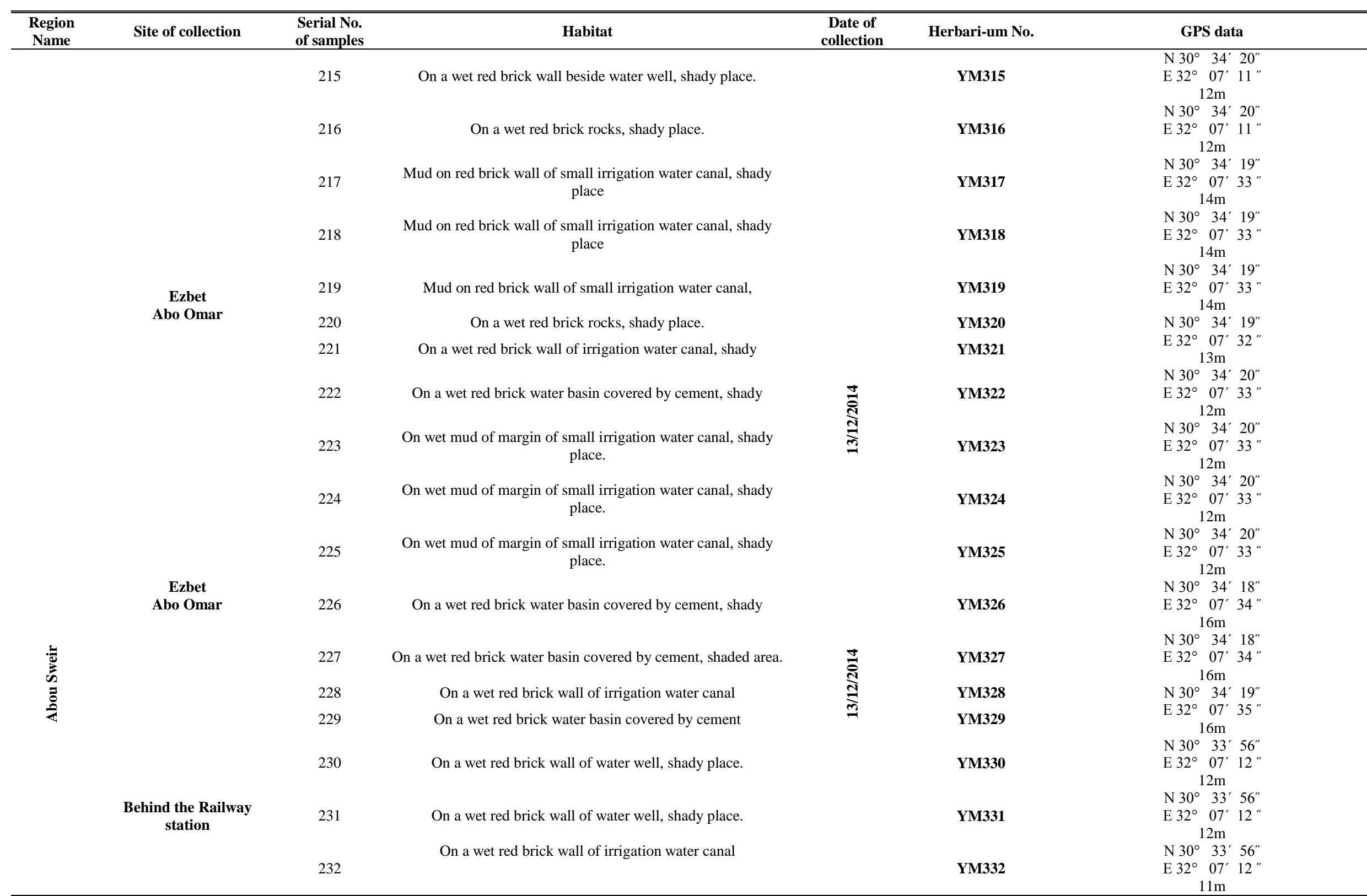




\section{Comparative Study of the Moss Flora}

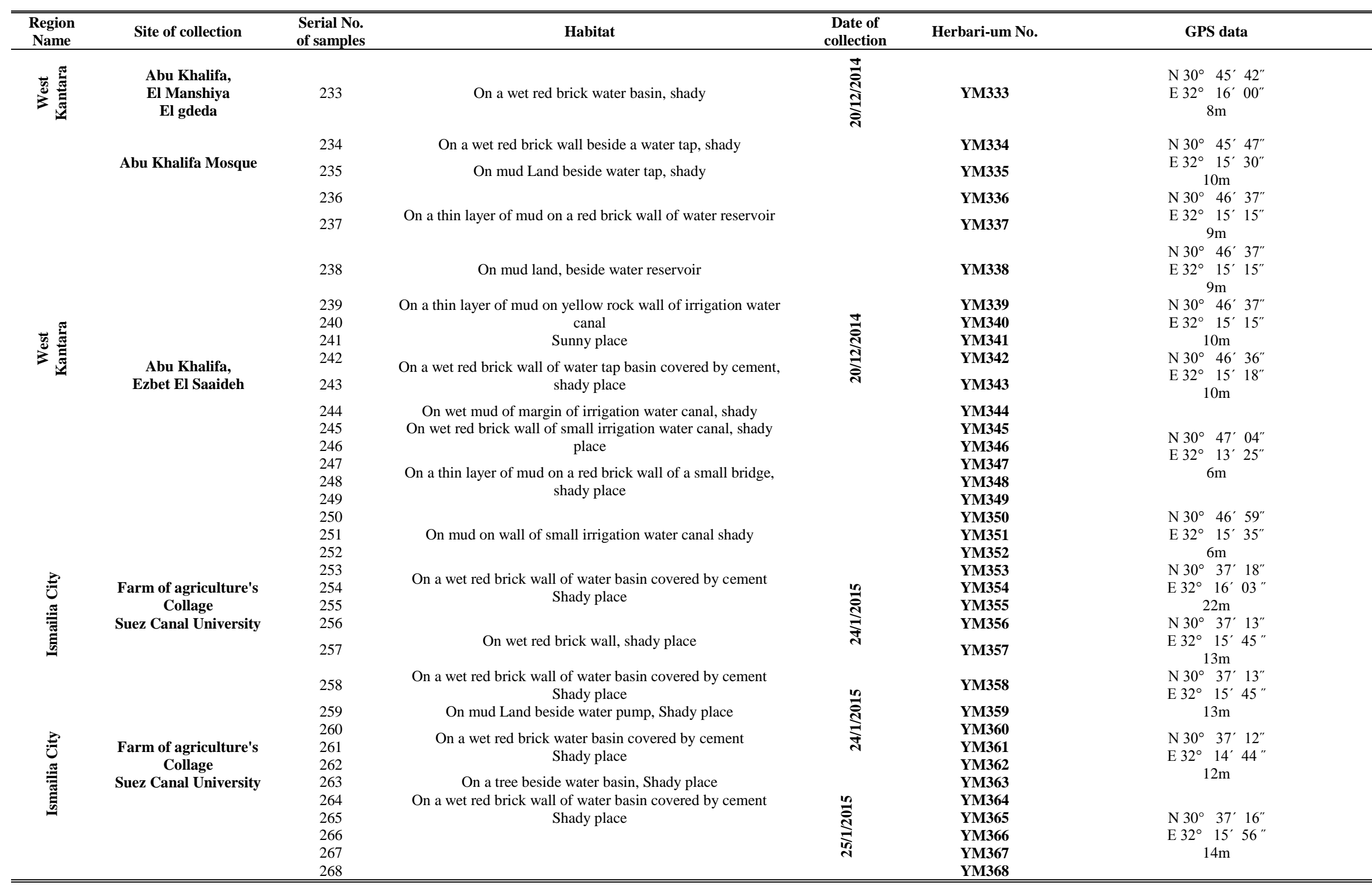




\section{Hassan et al.}

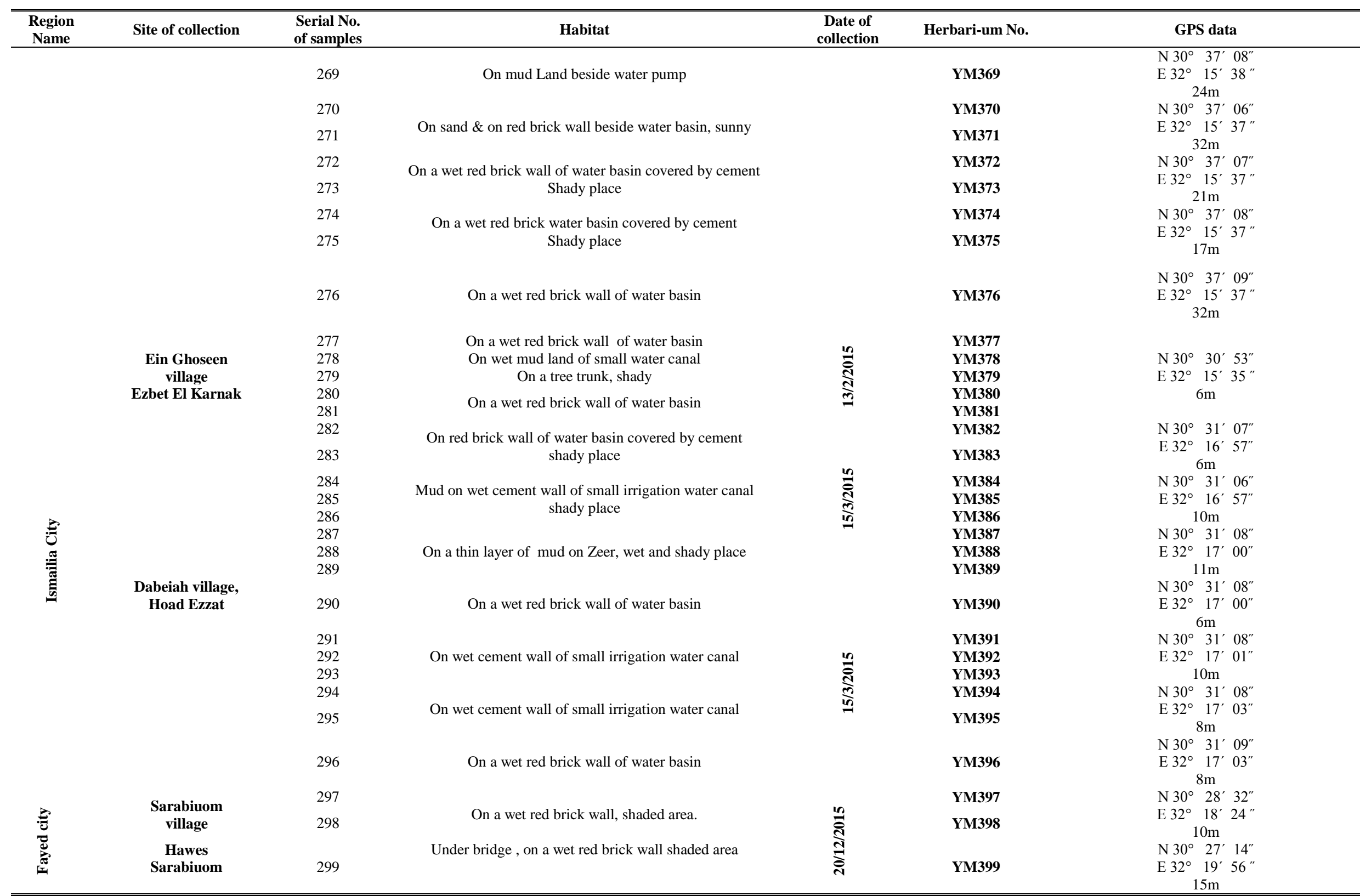




\section{Comparative Study of the Moss Flora}

\begin{tabular}{|c|c|c|c|c|c|c|}
\hline $\begin{array}{c}\text { Region } \\
\text { Name }\end{array}$ & Site of collection & $\begin{array}{c}\text { Serial No. } \\
\text { of samples }\end{array}$ & Habitat & $\begin{array}{c}\text { Date of } \\
\text { collection }\end{array}$ & Herbari-um No. & GPS data \\
\hline \multirow{11}{*}{ 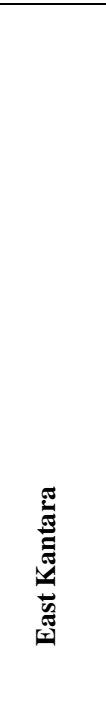 } & \multirow{5}{*}{$\begin{array}{l}\text { Abou Soultan } \\
\text { village, } \\
\text { Ezbet El Shahid } \\
\text { Ahmed Alqrali }\end{array}$} & 300 & \multirow{5}{*}{$\begin{array}{l}\text { Mud on wet cement wall of small irrigation water canal, shady } \\
\text { place }\end{array}$} & & YM400 & \multirow{2}{*}{ 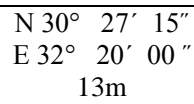 } \\
\hline & & 301 & & & YM401 & \\
\hline & & 302 & & & YM402 & $\mathrm{N} 30^{\circ} 25^{\prime} 15^{\prime \prime}$ \\
\hline & & 303 & & & YM403 & E $32^{\circ} 18^{\prime} 08^{\prime \prime}$ \\
\hline & & 304 & & & YM404 & $11 \mathrm{~m}$ \\
\hline & \multirow{2}{*}{$\begin{array}{l}\text { Ezbet } \\
\text { Al saadya }\end{array}$} & 305 & $\begin{array}{c}\text { On a thin layer of mud on a wall of water basin } \\
\text { Shady place }\end{array}$ & & YM405 & 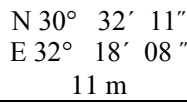 \\
\hline & & 306 & On a wet red brick wall of water basin shady place & & YM406 & $\begin{array}{ccc}\mathrm{N} 30^{\circ} & 32^{\prime} & 11^{\prime \prime} \\
\mathrm{E} 32^{\circ} & 29^{\prime} & 12^{\prime \prime} \\
& 15 \mathrm{~m}\end{array}$ \\
\hline & \multirow{4}{*}{$\begin{array}{l}\text { Eastern lakes, } \\
\text { El- ahwad area }\end{array}$} & 307 & On a wet red brick wall of water basin & \multirow{4}{*}{$\underset{\substack{\frac{\pi}{0} \\
\hdashline}}{\infty}$} & YM407 & $\mathrm{N} 30^{\circ} 32^{\prime} 04^{\prime \prime}$ \\
\hline & & 308 & On a wet red brick wall of water basin, shady place & & YM408 & $\begin{array}{c}\mathrm{E} 32^{\circ} 23^{\prime} 56^{\prime \prime} \\
19 \mathrm{~m}\end{array}$ \\
\hline & & 309 & On a wet red brick wall of water basin, shady place & & YM409 & $\begin{array}{ccc}\mathrm{N} 30^{\circ} & 32^{\prime} & 03^{\prime \prime} \\
\mathrm{E} 32^{\circ} & 23^{\prime} & 55^{\prime \prime} \\
27 \mathrm{~m}\end{array}$ \\
\hline & & 310 & On a wet red brick wall of water basin, shady place & & YM410 & $\begin{array}{l}\mathrm{N} 30^{\circ} 32^{\prime} \quad 00^{\prime \prime} \\
\mathrm{E} 32^{\circ} 23^{\prime} 59^{\prime \prime} \\
20 \mathrm{~m}\end{array}$ \\
\hline
\end{tabular}




\section{دراسة مقارنة فلورا الحزازيات بمحافظة الأسماعلية مع بعض المناطق الجغرافية الاخري في جمهورية

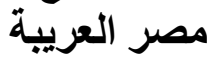

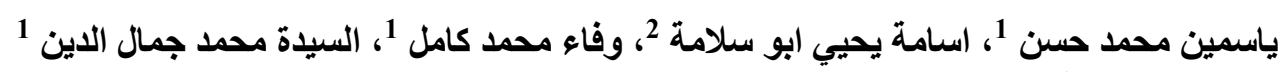

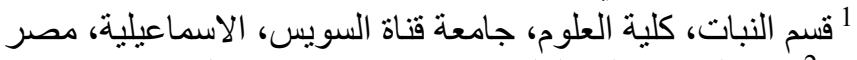

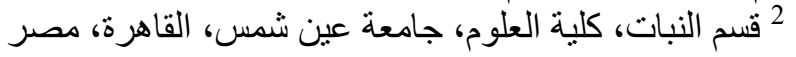

\section{الملخص العربي}

أجريت هذه الدراسة لمقارنة فلورا الحزازيات التي تم جمعها من محافظة الأسماعيلية بالفلورا الحزازية لباقي مناطق مصر الجغر افية. وتتبع أهمبة هذه الدر اسة من كونها دراسة حديثة لهذه المحافظة التي لم تشهد أي دراسة من هذا النوع منذ ثناثة عقود.

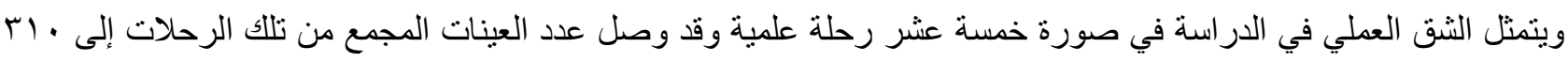

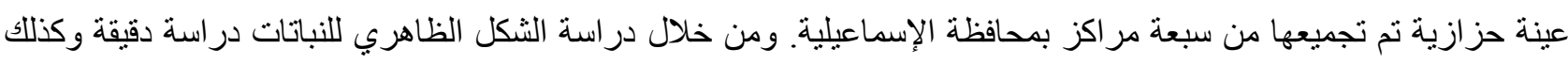
در استها تشريحيا و عمل مقاطع عرضية في الساق والأوراق لجميع العينات المدروسة. نم تعريف الأنواع المختلفة تعريفاً علمياً كاملاً عن طريق الاستعانة بمر اجع عديدة متخصصة ومزودة بصور فوتوغر افية ورسومات توضيحية. وقد اظهرت النتائج ان

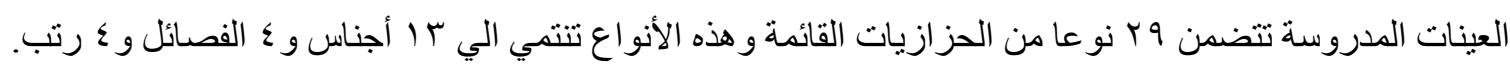

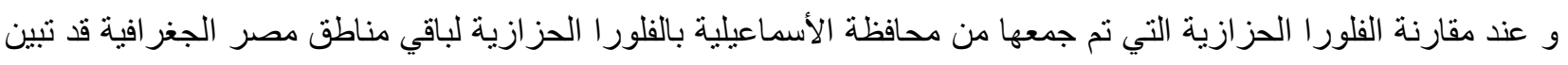
من ذلك ان: منطقة جنوب سيناء هي أكثر المناطق قربا من ناحية فلورا الحزازيات لمحافظة الأسماعيلية، بينما اقلها تشابهاً هما منطقتي نوبة النيل وجبل علبة. 